\title{
The Eroding Disk of AU Mic
}

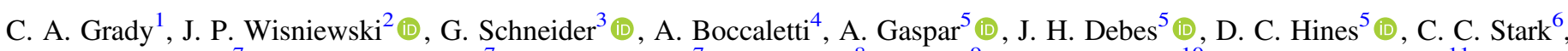 \\ C. Thalmann ${ }^{7}$ (D), A.-M. Lagrange ${ }^{7}$, J.-C. Augereau ${ }^{7}$, E. Sezestre ${ }^{8}$, J. Milli ${ }^{9}$ (D) , Th. Henning ${ }^{10}$, and M. J. Kuchner ${ }^{11}$ (i) \\ ${ }_{1}$ Eureka Scientific, 2452 Delmer Street, Suite 100, Oakland, CA 96402, USA \\ ${ }^{2}$ Homer L. Dodge Department of Physics and Astronomy, The University of Oklahoma, 440 West Brooks Street, Norman, OK 73019, USA \\ ${ }^{3}$ Steward Observatory, The University of Arizona, 933 North Cherry Avenue, Tucson, AZ 85721, USA \\ ${ }^{4}$ LESIA, Observatoire de Paris, Section de Meudon, 5, place Jules Janssen, F-92195 MEUDON Cedex, France \\ ${ }^{5}$ Lunar \& Planetary Laboratory, 1629 E. University Blvd, P.O. Box 210092, University of Arizona, Tucson, AZ 85721-0092, USA \\ ${ }^{6}$ AURA, STScI, 3700 San Martin Drive, Baltimore, MD 21218, USA \\ ${ }^{7}$ Institute for Particle Physics and Astrophysics, ETH Zurich, Wolfgang-Pauli-Strasse 27, 8093, Zurich, Switzerland \\ ${ }^{8}$ Univ. Grenoble Alpes, CNRS, IPAG, F-38000 Grenoble, France \\ ${ }^{9}$ ESO-Chile, Alonso de Cordova 3107 Vitacura-Santiago de Chile, Chile \\ ${ }^{10}$ Max Planck Institute for Astronomy, Koenigstuhl 17, D-69117 Heidelberg, Germany \\ ${ }^{11}$ NASA's GSFC, Exoplanets and Stellar Astrophysics Laboratory, Code 667, Goddard Space Flight Center, Greenbelt, MD 20771, USA \\ Received 2019 June 10; revised 2019 December 20; accepted 2019 December 21; published 2020 January 24
}

\begin{abstract}
We report Hubble Space Telescope (HST) Space Telescope Imaging Spectrograph imaging of AU Mic's debris disk from 2017 and archival data. Outward motion of the features in the southeast arm continues. At least three features have reached or exceeded projected escape velocity in the past decade, yielding a combined feature mass-loss rate of $\sim 1.2 \times 10^{-7} M_{\text {Earth }} \mathrm{yr}^{-1}$, or $\sim 1.2 \times 10^{-13} M_{\odot} \mathrm{yr}^{-1}, \sim 10 \%$ of AU Mic's stellar wind mass-loss rate, and similar to the ratio of coronal mass ejection mass loss to the stellar wind mass-loss rate. We confirm the 2018 finding of feature height changes for one feature $(B / \mathrm{SE} 4)$, but the $H S T$ data are too sparse to compare (yet) with the stellar activity cycle. Detection of what appears to be a chain of features in a second system suggests that the disk of AU Mic is not unique, although a larger sample of disks is required to quantify how common the phenomenon is.
\end{abstract}

Unified Astronomy Thesaurus concepts: Stellar activity (1580); Debris disks (363); M dwarf stars (982)

\section{Introduction}

Debris disks associated with solar-type stars have been imaged in scattered light in systems as old as $2.3 \mathrm{Gyr}$ (Schneider et al. 2016). Thus, our expectation was that M-star debris disks could be observable in scattered light over Gyr, because radiation pressure in these systems is ineffective in removing small grains (Matthews et al. 2015) although the stellar wind may displace material (Augereau \& Beust 2006). However, M stars with warm infrared (IR) excesses have proven rare after $30 \mathrm{Myr}$ (Binks \& Jeffries 2017, but see Zuckerman et al. 2019). Cold dust in older systems is detected in a few nearby systems (Lestrade et al. 2012; Kennedy et al. 2018), but has yet to be imaged in scattered light.

AU Mic (M1Ve, $V=8.63$ ), a member of the $\beta$ Pic Moving Group ( $t=24 \pm 3 \mathrm{Myr}$, Bell et al. 2015), at $d=9.72 \pm 0.04 \mathrm{pc}$ (Gaia Collaboration et al. 2018) hosts the best-studied M-star debris disk. It has a large IR excess $\left(L_{\mathrm{IR}} / L_{*}=0.44 \%\right.$, Schneider et al. 2014) from an edge-on debris disk (Kalas et al. 2004; Krist et al. 2005). The disk has a planetesimal belt at 35-40 au (Augereau \& Beust 2006; Strubbe \& Chiang 2006), similar in size to the solar system's Edgeworth-Kuiper Belt (MacGregor et al. 2013). The system has a halo (Matthews et al. 2015) extending to 130 au radius in scattered light (Schneider et al. 2014). The disk also has arc-shaped features, with projected sizes each of 5-10 au seen both in broad band optical Hubble Space Telescope (HST) images and near-infrared (NIR) Spectro-Polarimetric Highcontrast Exoplanet REsearch (SPHERE) data. The features move outward along the southeast (SE) arm of the disk (Boccaletti et al. 2015,2018 ), with projected velocities between 4 and $12 \mathrm{~km} \mathrm{~s}^{-1}$, and three of them have projected radial velocities above escape velocity. SPHERE observations (Boccaletti et al. 2018) showed broadening and blurring of the features over time. HST coronagraphic imagery offers the advantage of independent observing and data reduction strategies which conserve flux. We present $H S T$ images obtained in 2017 as well as earlier HST imagery.

\section{Observations}

\subsection{Space Telescope Imaging Spectrograph (STIS) Coronagraphic Imaging and Occulters Used}

Coronagraphic observations of $\mathrm{AU}$ Mic and point-spread function (PSF) template stars were obtained with the HST/ STIS in 2017-2018 as part of HST program GO-15219 (PI: J. Wisniewski), employing STIS's 50CORON imaging mode ${ }^{12}$ (Riley 2019; Section 12.11 therein). This mode provides a broad, unfiltered, spectral passband of pivot wavelength $0.575 \mu \mathrm{m}$ with $\mathrm{FWHM}=0.433 \mu \mathrm{m}$, an image scale of $50.077 \mathrm{mas}_{\text {pixel }}{ }^{-1}$ at the detector focal plane, and spatial resolution of $\sim 72$ mas. We used three different coronagraphic mask configurations: (1)—WedgeA0.6 (0." 3 half-width), (2)-WedgeA-1.0 (0." 5 half-width), and (3) -BAR5 (a 0!" 15 wide bar). The BAR5 data are discussed in Wisniewski et al. (2019). Comparable Wedge-A images of AU Mic were obtained as part of GO-12228 (Schneider et al. 2014, and Boccaletti et al. 2015).

\subsection{Three-roll Disk Imaging}

In Visits 01, 03, and 05 (2017 October 19-20) the AU Mic disk was imaged in three non-contiguous HST orbits, with interleaved PSF template star observations (Schneider et al. 2014, 2016). The spacecraft orientations were chosen so that disk images are unaffected by HST's unapodized diffraction spikes (see Figure 1).

\footnotetext{
$\overline{12}$ http://www.stsci.edu/hst/stis/documents / handbooks/currentIHB/stis_ ihb.pdf
} 


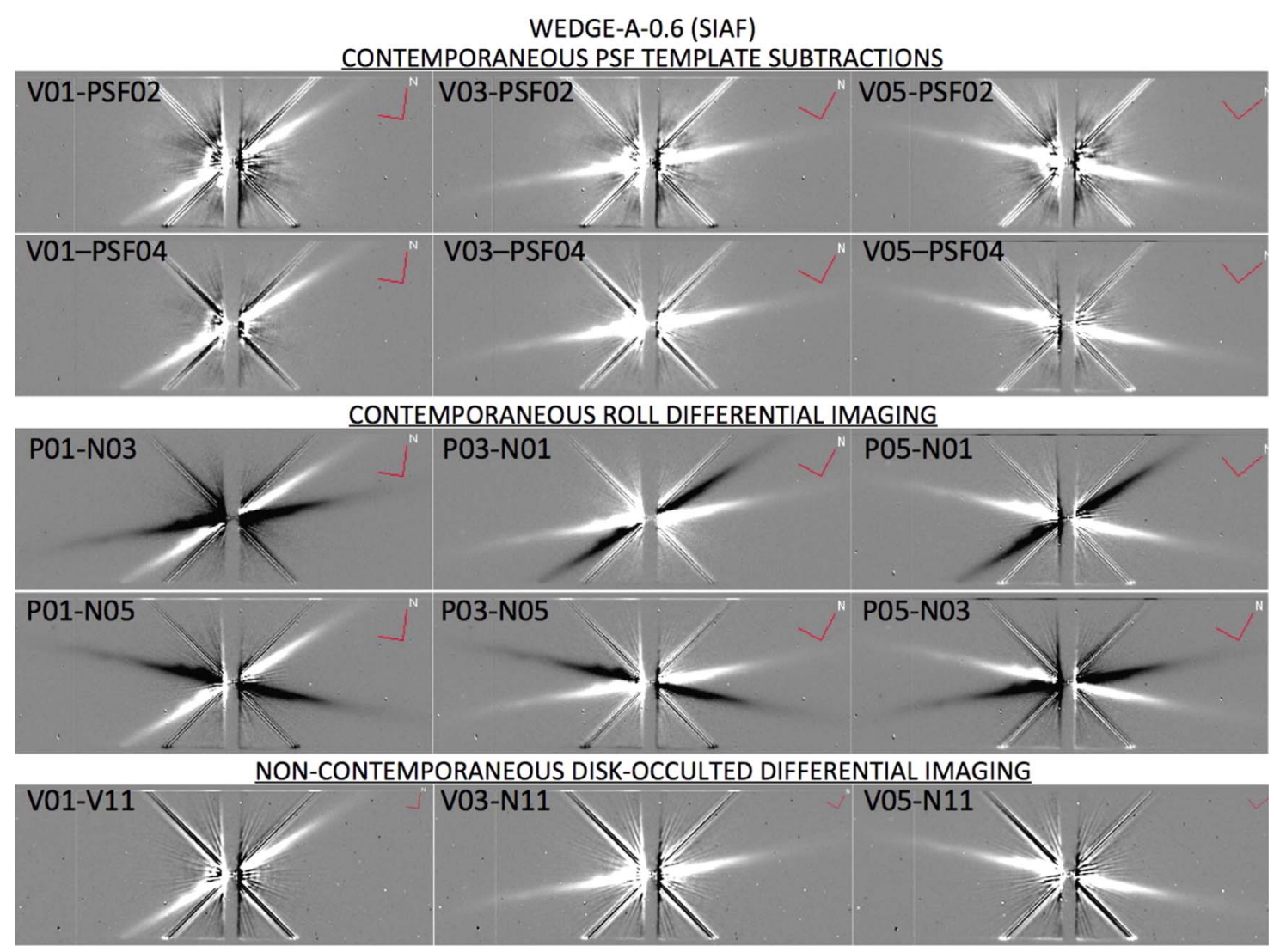

Figure 1. Comparison of three different PSF subtraction techniques applied to WedgeA-0.6 images. All panels 16 "! $96 \times 6$ "! 75 shown with a linear display from -2 (black) to +2 (white) counts s${ }^{-1}$ pixel $^{-1}$ (directions to north as indicated).

Visits 01 and 05 differ from Visit 03 in celestial orientation by $\pm 21^{\circ} .3$ to improve disk visibility, artifact rejection, and PSF sampling when combined following PSF subtraction. In each visit, multiple WedgeA-0.6 and then three WedgeA-1.0 exposures were obtained. Exposure durations for WedgeA0.6 reach, without saturation, $80 \%$ full well depth for pixels at the coronagraphic inner working angle immediately beyond the edge of the occulting wedge. To provide higher signal-to-noise ratio $(\mathrm{S} / \mathrm{N})$ at larger stellocentric angle, the WedgeA-1.0 exposures were saturated immediately beyond the occulting wedge edge. This region was well covered in the WedgeA-0.6 imaging, and later combined in post-processing. Due to $H S T$ guide star acquisition performance issues at the time of observation, a few of the planned exposures in each visit were unstable or failed to execute. For Visits 01 and 03, the WedgeA-0.6 total integration times were reduced from a planned $546 \mathrm{~s}$ to $364 \mathrm{~s}$, and $483 \mathrm{~s}$, respectively, and for Visit 05 the WedgeA-1.0 total integration time was reduced from a planned $1592 \mathrm{~s}$ to $1061 \mathrm{~s}$.

\subsection{Contemporaneously Imaged Reference Stars}

In Visits 02 and 04 we obtained coronagraphic images of two PSF template stars. Both have optical color indices very close to AU Mic, and are in close proximity on the sky. Both were observed in the same manner as the AU Mic disk, but only at the HST-nominal celestial orientation angle for each. These two visits were interleaved contiguously between AU Mic visits 01/03 and 03/05.The PSF template star in Visit 02 was HD $198939(V=7.97 ; \Delta[B-V]$ w.r.t. AU Mic $=+0.01$; exp. times: WedgeA-0.6 $=16 \times 20.7 \mathrm{~s}$, WedgeA-1.0 $=4 \times 377.0 \mathrm{~s}$ ), and in Visit 04 was HD $191849(V=7.66 \Delta[B-V]$ w.r.t. $\mathrm{AU}$ Mic $=-0.02$; exp. times: WedgeA-0.6 $=16 \times 30.6 \mathrm{~s}$, WedgeA-1.0 $=4 \times 416.5 \mathrm{~s}$ ).

\subsection{Disk-occulted Imaging}

"Roll differential Imaging" (RoDI), as enabled by observations such as those described in Section 2.2 for WedgeA with sufficiently large differential rolls, is an effective method for point-source imaging to obviate chromatic residuals that otherwise arise from imperfectly matched target:template spectral energy distributions (SEDs) with PSF template subtraction. The same is true for edge-on disks, like $\mathrm{AU}$ Mic, where the degree of differential rotation exceeds the angular distance in separation of the positive and negative imprints of the disk in difference images. Larger roll angles probe to smaller effective inner working angles (IWAs). Ideally, $90^{\circ}$ of differential rotation would be optimal, but such large rolls at a single epoch exceed HST operational constraints. In single-orbit Visit 11 (WedgeA, 2018 July 24 UT), we imaged AU Mic with the edge-on disk close to orthogonal to the Visit 03 and a series of 23 images taken and with the disk occulted behind the long axis of WedgeA. Other than differences in exposure times (WedgeA-0.6: $8 \times 45.5 \mathrm{~s}, 8 \times 22.7 \mathrm{~s}$; WedgeA-1.0: $3 \times 500 \mathrm{~s}$ ) imaging was as described in Section 2.2. These images provide perfectly color-matched PSF templates for subtraction potentially enabling the smallest possible RoDI IWAs. However, when scheduled non-contemporaneously other systematics due to PSF instability over time may dominate. 


\section{Calibration, Reduction, and PSF Subtraction}

For image calibration, reduction, and PSF subtraction, we outline below the methods and procedures adopted (Schneider et al. 2014, 2016, 2017, 2018).

\subsection{Instrumental Calibration and Image "Visit-level" Reduction}

We closely followed the successful observational paradigm for HST multi-roll PSF-template subtraction coronagraphy as demonstrated in HST GO program 12228 (Schneider et al. 2014) and detailed by Schneider et al. (2016). After converting to count rates, the individual images were vetted against anomalous pointing drifts, and execution incompleteness. Such images, when found (see Section 2.2 for WedgeA) were rejected from further reduction. In Visit 11 (only) exposure-toexposure pointing stability was degraded by a 41 mas rms lineof-sight jitter about an otherwise stable image centroid position. This was partially mitigated through digital image resampling (see below), though coronagraphic image contrast near the IWA was somewhat compromised w.r.t. nominal performance. Within each visit, all images observed with the same occulter and target position (i.e., WedgeA location) were then median combined to form "visit level," S/N-improved, count rate images also mostly cleansed of cosmic-ray hits and other transient read-out artifacts. Inter-visit image co-registration of the visit-level images (and later PSF subtractions) were done with sinc-apodized bi-cubic sub-pixel interpolation using the IDL-based, i $d p 3^{13} \mathrm{~S} / \mathrm{W}$ (Stobie \& Ferro 2006).

We prepared PSF-subtracted images using each PSF subtraction technique (Sections 2.2-2.4) at the visit level. Template (only) relative brightness and $(X, Y)$ positions were treated, and iteratively adjusted, as free parameters to minimize the variance in difference image pixels in the target regions of interest not dominated by disk flux. In doing so, a priori known bad pixels, areas affected by the imprints of the image plane occulter, HST diffraction spikes, regions of image saturation, and other know image artifacts were excluded.

\subsection{Visit-level PSF Subtractions and Three-orientation Image Combination}

For each visit-level disk image we applied three methods of reference PSF subtraction ((a)-(c) below) from disk image data acquired for this purpose at three field orientations with all three occulter configurations using: (a) PSF-template subtraction with two calibration/reference stars, (b) coronagraphic RoDI for the WedgeA visits, and (c) "self-subtraction" using AU Mic as its own PSF template with the disk obscured by the chosen image plane occulter. In Figure 1 we representatively illustrate, and inter-compare, the results from these three methods for the WedgeA- 0.6 occulter.

The top two sets of panels in Figure 1 show the results of (a) PSF template subtraction with the disk at three orientation angles changing in the instrument frame incrementally by 21.3 from Visit 01, to 03, to 05. The top row uses HD 198939 (designated "PSF02") as a template star, and the second row uses HD 191849 (designated "PSF04"). While both reference stars have $B-V$ color indices nearly identical to AU Mic, the use of PSF02 resulted in much larger amplitude chromatic image artifacts (dark and light "halos") that are easily seen circumscribing the bright midplane of

\footnotetext{
${ }^{13}$ https://archive.stsci.edu/prepds/laplace/idp3.html
}

the edge-on disk. With the use of PSF04 most of these residuals (that are discussed in detail by Schneider et al. (2014; Section A.10 and Figures 36 and 37 therein) are eliminated, but a narrow chromatic ring at $r \sim 0$ !" 8 (more readily seen on the SE (left) side of the star in the V01-PSF04 subtraction) remains. This artifact is largely correctable following Schneider et al (2014, Section A.3), and is fully eliminated with either RoDI or diskocculted PSF subtractions.

In the middle panels, the results of (b) RoDI with three independent images (one at each roll for each Wedge) are shown in both positive $(\mathrm{P})$ and negative $(\mathrm{N})$ imprint parities with chromatic residuals fully obviated. The roll differentials are sufficiently large that disk sub-structures both above and below the disk plane are unambiguously revealed nearly to the IWA imposed by the Wedge edges or (for WedgeA-1.0) regions of inner saturation. In particular, in P05-N01, the positive image imprint of the out-of-plane "bump" at the top of the innermost disk feature (designated "A") and its complementary (negative) inverse are separated by $\sim 1$ ". 07 , whereas the half-height of the out-of-plane vertical structure is $\sim 0$ !" 33 .

The bottom panels in Figure 1 show the result of (c) subtracting AU Mic's co-registered stellar PSF with the disk itself obscured by Wedge-A in the reference image. Here, chromatic residuals are fully mitigated, but due to the non-contemporaneous nature of the observations, PSF-subtraction residuals due to differential wavefront errors (mostly focus terms) manifest as "tendrils" radiating from the location of the star.

For each PSF-subtraction technique, the images are reoriented to a common (north being "up") celestial orientation. Digital masks, unique to each image, are then created to reject data containing or affected by: (1) disk negative imprints in the case of RoDI, (2) the imprint of the occulter used, (3) the HST diffraction spikes, and (4) saturated or otherwise defective pixels. Maskedmedian combinations of those celestially reoriented images then reveal the global disk structure, minimizing unsampled areas. We illustrate the results, with the so-treated three-roll combined WedgeA-0.6 RoDI imaging, and follow Schneider et al. (2014) in defining the disk midplane in Figure 2.

\section{Imaging Results}

\subsection{Disk Structure Temporal Evolution}

STIS multi-roll PSF-subtracted coronagraphic imaging of the AU Mic debris disk over $\sim 7.19 \mathrm{yr}$ is shown in Figures 3 and 4. The STIS epochs all show out-of-midplane structures, which are more conspicuous in the SE arm (Figure 3), but also seen in the northwest (NW) arm (Figure 4). The data from 2010 to 2011 were initially combined and reported as a single intermediate epoch (Schneider et al. 2014), but reported separately by Boccaletti et al. (2015), and in this study. SPHERE data from 2014 to 2017 are discussed in Boccaletti et al. (2018). After scaling the surface brightness (SB) of the disk to compensate for the radial drop in the stellar illumination Figures (2)-(4)), the epochal locations and estimates of uncertainties of the individual features, identified by eye, were determined with idp3's photocentric 2D weightedmoment algorithm. ${ }^{14}$

\footnotetext{
${ }^{14}$ IDP3 (URL given in footnote 13) uses the "ASTRON Library" of IDL procedures maintained by the NASA Goddard Space Flight Center and may be downloaded through the IDL Astronomy User's Library (at website idlastro. gsfc.nasa.gov, or via the idp web page). For these measurements, idp3 implements the 1997 September version of the ASTRON idl_phot library routine CNTRD.pro. This is locally maintained in the idp3 package under the name idp3_CNTRD.pro.
} 


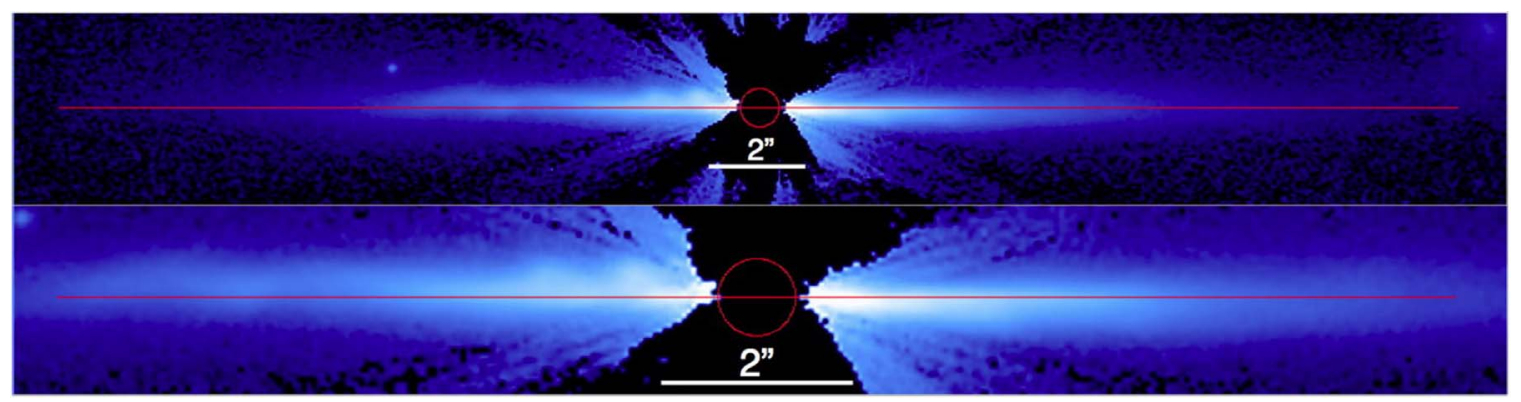

Figure 2. Three-RoDI combined images of the AU Mic disk with the disk midplane (SE side is left) on the image horizontal scaled by $r^{2}$ to compensate for illumination drop-off with stellocentric distance. The images are $\log _{10}$ displayed from [-3.5] to [+1.0] $\{\mathrm{dex}\}$ counts s$^{-1}$ pixel $^{-1}$. Top: field of view $31^{\prime \prime}$. $50 \times 4{ }^{\prime \prime}$. 10 , tracing starlight-scattering debris to $r \sim 150$ au along the disk plane. Bottom: field of view 15 " $75 \times 2$ " 05 , focusing on disk sub-structure at $r<75$ au to an effective IWA of $r=0$ ". 4 (3.9 au; indicated by the red circle) along the disk midplane.
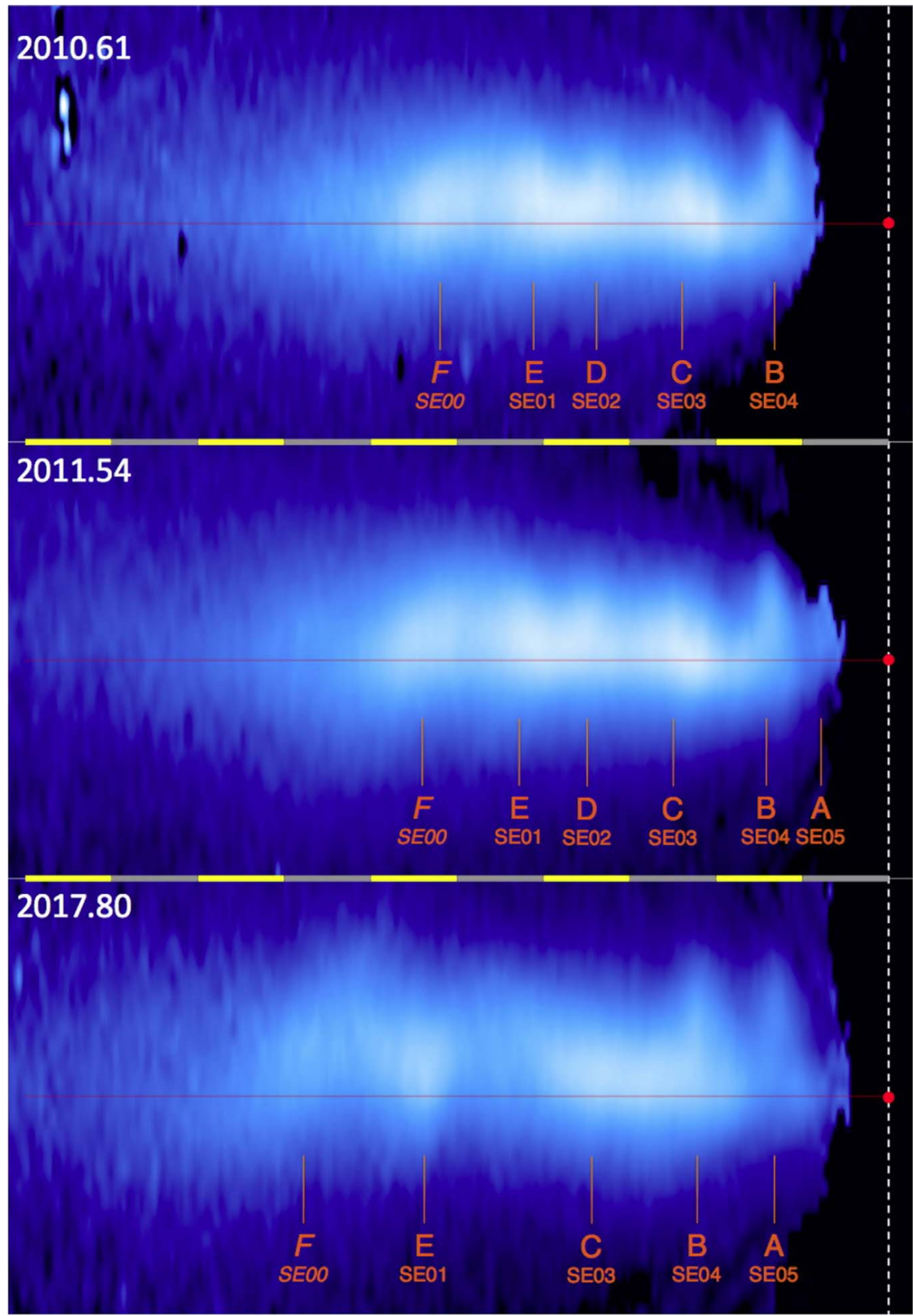

Figure 3. Changes in the locations, morphologies, and vertical extensions of the AU Mic moving features since the 2010 are striking. Features are identified following Boccaletti et al. (2015) and with nomenclature from Boccaletti et al. (2018) shown below. An additional feature beyond the SPHERE field of view is designated $F /$ SE00. One feature ( $D /$ SE02) could not be identified in the 2017 HST data. Features $A$ and $B$ are resolved in 2017, while features $E$ and $F$ are now extremely broad. Shown here is the SE side of the disk interior to 9"! 44 (92.4 au), with a $4 \times$ expansion in vertical spatial scale and scaling as for Figure 2 . The yellow/grays scale bars alternate with $1^{\prime \prime}$ spacings. 

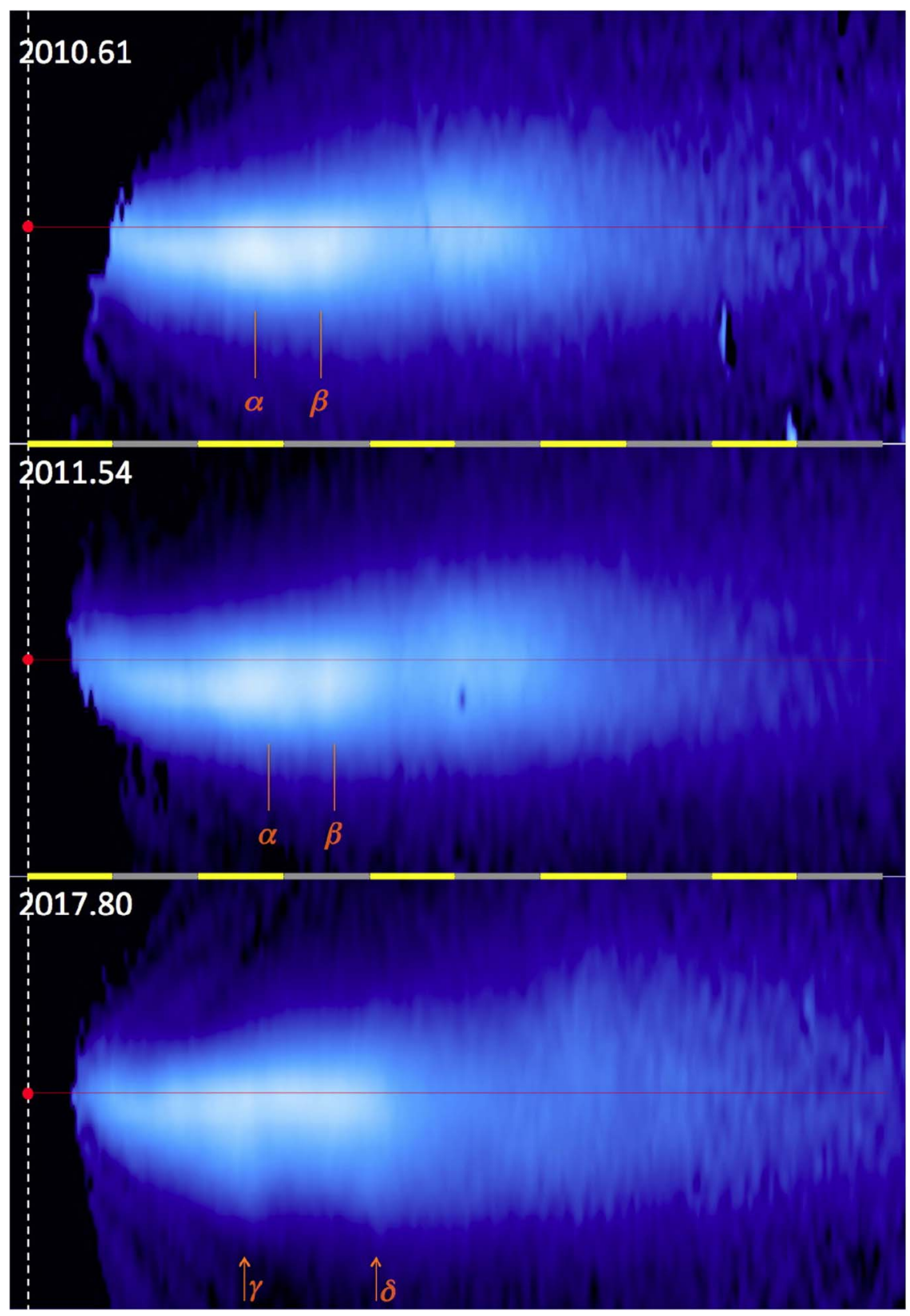

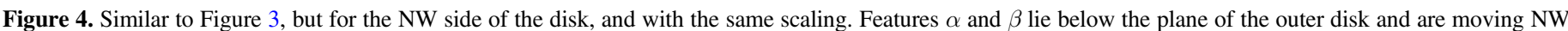

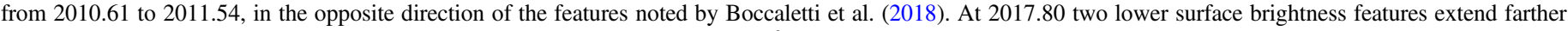
"below" the disk plane, indicated by the orange arrows and identified as $\gamma$ and $\delta$.

We confirm continued outward motion of the Boccaletti et al. (2015) features (Table 1), as well as broadening and morphological evolution of many of the features. The situation in the NW arm is more complex. Features $\alpha$ and $\beta$ (Figure 4) extend below the disk midplane. Both move to the NW from 2010 to 2011 at with projected velocities over $2010-2011$ of $7.43 \pm 3.51 \mathrm{~km} \mathrm{~s}^{-1}$ for $\alpha$ and $8.43 \pm 3.51 \mathrm{~km} \mathrm{~s}^{-1}$ for $\beta$. Feature $\beta$ may be escaping. If we assume constant outward velocities from 2011 to 2017 , the features should be near 3!" $8(\alpha)$ and 4 !. $7(\beta)$ for the 2017.8 observation. In 2017, two features (working identifications $\gamma$ and $\delta$ ) extend farther below the disk midplane with one at 2 ". $54 \pm 0$ ". 09 , and the other at 4 ". $07 \pm 0$ ". 10 . Neither NW feature coincides with the extrapolated locations of $\alpha$ or $\beta$, which may indicate that the motion is not constant projected velocity that we have not recovered the previously identified features, or that the assumption of continued outward motion is incorrect. The STIS NW feature motions differ from the SPHERE NW features in direction of motion (Boccaletti et al. 2018). If all features rotate in the disk in the same sense, the detection of features in the NW arm moving in different directions can be reconciled if we have detected features on the near and far sides of the disk.

Four features (Table 1) can be tracked for longer than $7 \mathrm{yr}$. Measured projected velocities range between $-5.74 \pm 0.075$ $(B / S E 4),-5.04 \pm 0.93(C / S E 3),-8.20 \pm 2.84(E / S E 1)$ and $-10.27 \pm 3.72(F / \mathrm{SE} 0) \mathrm{km} \mathrm{s}^{-1}$. In our data, two features, $E / \mathrm{SE} 1$ and $F / \mathrm{SE} 0$, exceed escape velocity for masses for AU Mic ranging from 0.4 to $0.8 M_{\odot}$. Boccaletti et al. (2018) identified an additional feature, D/SE2, as escaping. This feature's linearly extrapolated location corresponds to a gap at the disk midplane in the 2017 HST data. We conclude that at least three features have 


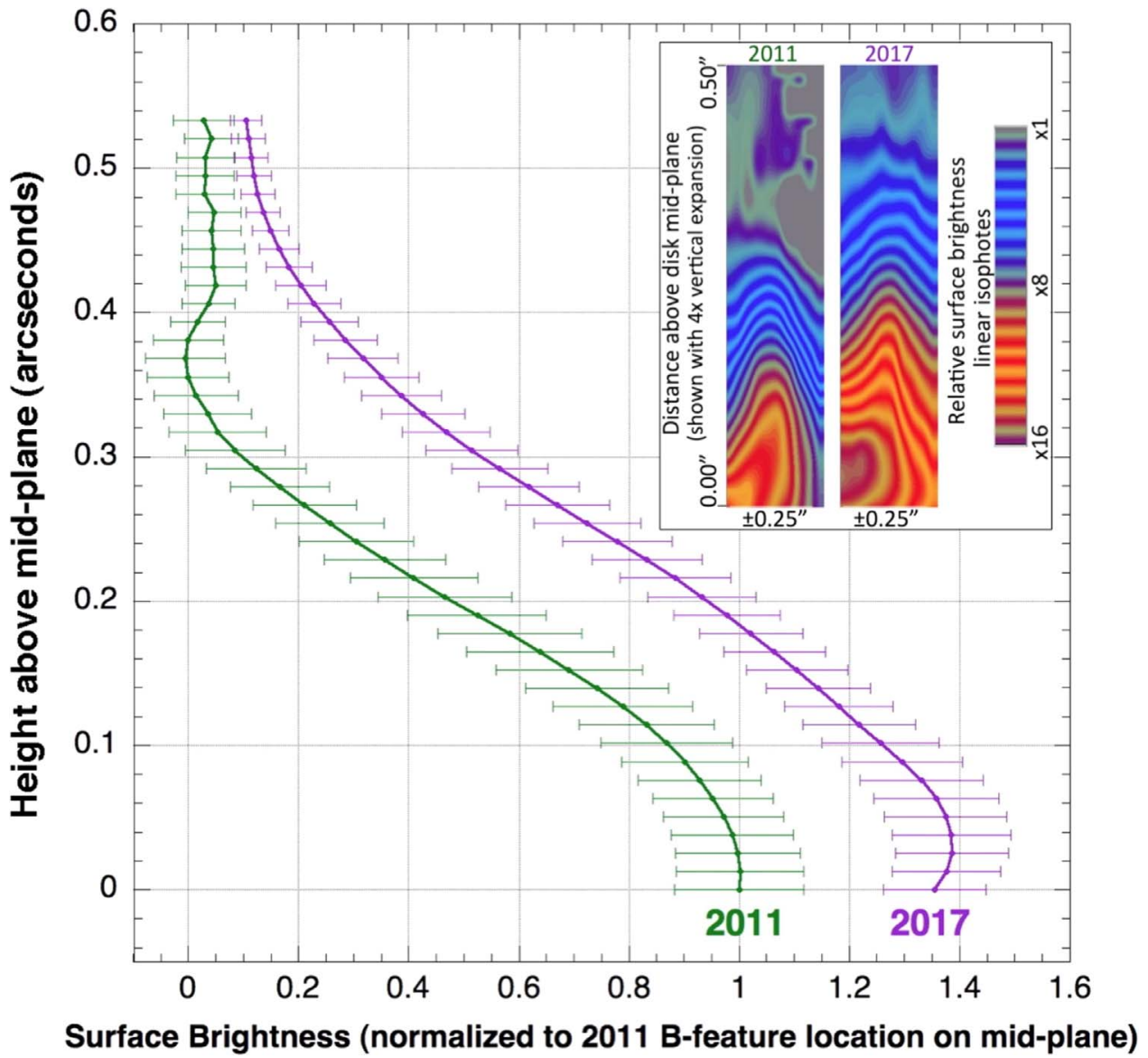

Figure 5. Relative changes in feature B SB (as first-order proxy to dust density) and vertical height above disk midplane from epoch 2011 to 2017 . SB normalized to 2011 epoch on midplane. PLOT: Lines-total flux in 0.15 (10 pixel) wide 1D cross-sectional measures (a.k.a. "slices") at each vertical location in the $2 \mathrm{D}$ images above disk midplane. Bars-variance along each slice as proxy to feature width. INSET: SB isophote contours increment linearly by $6.25 \%$ sequentially peak-to-peak over full (16:1) dynamic range. The vertical region shown is $0 . " 5$ for both data sets.

Table 1

Feature Locations ${ }^{\mathrm{a}}$

\begin{tabular}{|c|c|c|c|c|c|c|c|c|}
\hline $\mathrm{EPOCH}$ & $F$ & $E$ & $D$ & $C$ & $B$ & $A$ & $\alpha$ & $\beta$ \\
\hline 2010.61 & -5 !" 19 & $-4 ! " 11$ & -3 "! 39 & $-2 ! " 39$ & -1 !" 32 & $\mathrm{~N} / \mathrm{A}$ & $+2 ! " 67$ & $+3 \prime \prime 42$ \\
\hline 2011.54 & $-5 ! 40$ & $-4 ! .29$ & $-3 " .49$ & $-2 ! 50$ & $-1 " .41$ & $-0 ! " 79^{b}$ & $+2{ }^{\prime \prime} 82$ & +3 ". 59 \\
\hline 2017.80 & $-6 ! 78$ & $-5 !$ ! 38 & $\ldots^{c}$ & $-3 ! 117$ & $-2 ! 221$ & -1 ". 32 & $\ldots^{c}$ & $\ldots^{c}$ \\
\hline
\end{tabular}

Notes.

${ }^{a}$ Stellocentric angular distance along disk midplane; negative values to SE, positive values to NW of AU Mic, positions are uncertain to \pm 0 !. 05 for $A, B, \alpha$ and $\beta$ and 0 !" 1 for $C, 0$." 2 for $E$, and 0 !. 4 for F, reflecting the more diffuse nature of features $C-F$. Features $\gamma$ and $\delta$ are seen in 2017 and will be discussed with new HST observations.

${ }^{\mathrm{b}} \mathrm{A}(2011.54)$ feature is partially obscured by IWA-may be negatively biased in location.

c ... Indicates feature undetected or ambiguous in 2017 HST data.

projected velocities above escape velocity. Monitoring over the next few years (now in progress) should enable us to determine whether additional features are escaping.

\subsection{Implications for Modeling the Features}

Two recent models have been proposed for AU Mic's moving features. The first invokes dust avalanches at the intersection of an out-of-plane belt, produced by the destruction of a Varuna-sized body $(r=668 \mathrm{~km}$, Santos-Sanz et al. 2013) with the main planetesimal belt (Chiang \& Fung 2017). Unlike $\beta$ Pic (Krist et al. 2005), we do not find an out-of-plane belt in the HST data following subtraction of a model outer disk. An in-plane, eccentric dust belt is possible, but would be hard to separate from the edgeon disk. This model was designed to produce features only in the $\mathrm{SE}$ arm of the disk, but if the location of the belt intersection is moved, it could produce features in the NW.

Chiang \& Fung (2017) predicted that the feature elevations should oscillate with the period of a stellar activity cycle. Ibañez Bustos et al. (2019) report a 5 yr chromospheric activity cycle for AU Mic. The data presented in Boccaletti et al. (2018) show elevation changes on timescales of several months rather than a $5 \mathrm{yr}$ period. We confirm height changes for one feature (B/SE4) from 2011 to 2017 (Figure 5), but the HST data are 
too sparse for period fitting. Alternatively, Sezestre et al. (2017) included models for features launched from either a stationary location at $r=27 \mathrm{au}$, or at a planet orbiting the star at $8 \pm 2$ au. Continued observations that track the evolution of feature positions and SBs will help discriminate between the suggested models in the literature.

With a mass per feature of $4 \times 10^{-7} M_{\text {Earth }}$ (Chiang \& Fung 2017), and loss of three features per decade, the mass-loss rate is $1.2 \times 10^{-7} M_{\text {Earth }} \mathrm{yr}^{-1}\left(1.2 \times 10^{-13} M_{\odot} \mathrm{yr}^{-1}\right)$. If the stellar wind mass-loss rate for AU Mic is $\sim 50 \times$ that of the modern Sun (Schüppler et al. 2015), the ratio of feature-tostellar-wind mass loss is $\sim 0.12$, similar to the ratio of coronal mass ejection (CME) mass-loss rate to solar wind mass-loss rate (Mishra et al. 2019). Analysis of the 2018 HST BAR5 and Transiting Exoplanet Survey Satellite (TESS) data suggests potential sculpting of the disk by a differential stellar wind (Wisniewski et al. 2019). Collectively, these findings suggest that the stellar wind and CMEs should be further explored for a causal role in feature motion and ejection.

\subsection{Is AU Mic Typical?}

AU Mic is noted in the literature for youth, proximity to Earth, rapid stellar rotation and high stellar activity levels, and youth, as well as the features in its disk that have been observed since 2004. Other M-star members of the $\beta$ Pic-AB Dor Moving Groups have similar $L x / L_{\mathrm{Bol}}$ (Kastner et al. 2011) and rapid rotation (Binks \& Jeffries 2016) to AU Mic, implying similar stellar radiation fields. These findings suggest that AU Mic as a young star is notable only for proximity.

AU Mic hosts one of four debris disks hosted by M-stars that are imaged to date in scattered light (the others are TWA 7 and 25; see Choquet et al. 2016 and GSC 07396-00759; see Sissa et al. 2018). GSC 07396-00759 may also be a member of the $\beta$ Pic Moving Group (Kastner et al. 2011) with the same age as AU Mic. It is viewed at $i=83^{\circ}$ from pole-on. The disk has "ripples" along one side, and is described similarly to early studies of AU Mic. Detection of what appears to be a chain of features in that system suggests that the disk of AU Mic is not unique, and that ejection of features may be common in early M stars, although a larger sample of stars is required to quantify this. We conclude that AU Mic appears to be a typical, young early M-star with a debris disk whose structure and longevity may be influenced by its stellar host.

\section{Summary}

Outward motion of the features identified in AU Mic's disk by Boccaletti et al. (2015) continues. We confirm the presence of features in the NW arm, finding two features in the NW arm in 2017 data, and two in earlier data. The features detected in 2010-2011 move to the NW, unlike the close-in pair detected by Boccaletti et al. (2018) moving to the SE. They may be seen on opposite sides of the disk. We find the mass-loss rate by features is $1.2 \times 10^{-13} M_{\odot} \mathrm{yr}^{-1}, \sim 10 \%$ of the estimated stellar wind mass-loss rate, and similar to the ratio of mass loss by CMEs and the wind in the solar system.
This may indicate a link between feature mass loss and CMEs (Wisniewski et al. 2019).

This study was supported by and the new HST observations obtained under HST-GO-15219 which also supported C.A.G., G.S., and J.P.W., using the NASA/ESA Hubble Space Telescope. Earlier data were obtained from the archive at the Space Telescope Science Institute. STScI is operated by the Association of Universities for Research in Astronomy, Inc. under NASA contract NAS 5-26555. J.C.A. and E.S. acknowledge financial support from the Programme National de Planétologie (PNP) of CNRS-INSU co-funded by the CNES. We wish to thank the referees for useful suggestions.

\section{ORCID iDs}

J. P. Wisniewski (iD https://orcid.org/0000-0001-9209-1808

G. Schneider (iD https://orcid.org/0000-0002-4511-5966

A. Gaspar (1) https://orcid.org/0000-0001-8612-3236

J. H. Debes (ib https://orcid.org/0000-0002-1783-8817

D. C. Hines (ib https://orcid.org/0000-0003-4653-6161

C. Thalmann (iD https://orcid.org/0000-0002-1664-2177

J. Milli (i) https://orcid.org/0000-0001-9325-2511

M. J. Kuchner (ib https://orcid.org/0000-0002-9622-9605

\section{References}

Augereau, J.-C., \& Beust, H. 2006, A\&A, 455, 987

Bell, C. P. M., Mamajek, E. E., \& Naylor, T. 2015, MNRAS, 454, 593

Binks, A. S., \& Jeffries, R. D. 2016, MNRAS, 455, 3345

Binks, A. S., \& Jeffries, R. D. 2017, MNRAS, 469, 579

Boccaletti, A., Sezestre, E., Lagrange, A.-M., et al. 2015, Natur, 526, 230

Boccaletti, A., Thalmann, C., Lagrange, A.-M., et al. 2018, A\&A, 614, 52

Chiang, E., \& Fung, J. 2017, ApJ, 848, 4

Choquet, E., Perrin, M., Chen, C. H., et al. 2016, ApJL, 817, L2

Gaia Collaboration, Brown, A. G. A., Vallenari, A., et al. 2018, A\&A, 616, A1 Ibañez Bustos, R. V., Buccino, A. P., Flores, M., et al. 2019, MNRAS, 483, 1159

Kalas, P., Liu, M., \& Matthews, B. C. 2004, Sci, 303, 1990

Kastner, J. H., Sacco, G. G., Montez, R., et al. 2011, ApJL, 740, L17

Kennedy, G. M., Bryden, G., Ardila, D., et al. 2018, MNRAS, 476, 4584

Krist, J. E., Ardila, D. R., Golimowski, D. A., et al. 2005, AJ, 129, 1008

Lestrade, J.-F., Matthews, B. C., Sibthorpe, B., et al. 2012, A\&A, 548, A86

MacGregor, M. A., Wilner, D. J., Rosenfeld, K. A., et al. 2013, ApJL, 762, L21

Matthews, B. C., Kennedy, G., Sibthorpe, B., et al. 2015, ApJ, 811, 100

Mishra, W., Srivastava, N., Wang, Y., et al. 2019, MNRAS, 486, 4671

Riley, A. 2019, STIS Instrument Handbook, Version 18.0 (Baltimore, MD: STScI)

Santos-Sanz, P., Ortiz, J. L., Leilouch, E., et al. 2013, EPSC, 8, 665

Schneider, G., Debes, J., Grady, C. A., et al. 2018, AJ, 155, 77

Schneider, G., Gaspar, A., \& Debes, J. 2017, Enabling Narrow(est) IWA Coronagraphy with STIS BAR5 and BAR10 Occulters, Instrument Science Report, STIS 2017-03

Schneider, G., Grady, C. A., Hines, D. C., et al. 2014, AJ, 148, 59

Schneider, G., Grady, C. A., Stark, C. C., et al. 2016, AJ, 152, 64

Schüppler, Ch., Löhne, T., Krivov, A. V., et al. 2015, A\&A, 581, A97

Sezestre, E., Augereau, J.-C., Boccaletti, A., \& Thebault, P. 2017, A\&A, 607, A65

Sissa, E., Oloffson, J., Vigan, A., et al. 2018, A\&A, 613, L6

Stobie, E., \& Ferro, A. 2006, ASPC, 351, 540

Strubbe, L. E., \& Chiang, E. 2006, ApJ, 658, 652

Wisniewski, J. P., Kowalski, A. F., Davenport, J. R. A., et al. 2019, ApJL, 883, L8

Zuckerman, B., Klein, B., \& Kastner, J. 2019, ApJ, 887, 87 